\title{
Application of a Standard Operating Procedure in Transporting Patients during the COVID-19 Pandemic in the Air Emergency Medical Services
}

\author{
Mohammad Hossein Esmaeilzadeh; ${ }^{1,2}$ Mahmoudreza Peyravi; ${ }^{3} \odot$ Milad Ahmadi Marzaleh ${ }^{3,4,5} \odot$
}

1. PhD Student of Health in Disaster and Emergencies, Department of Health in Disasters and Emergencies, School of Management and Medical Information, Iran University of Medical Sciences, Tehran, Iran

2. Social Determinants of Health Research Center, Gonabad University of Medical Sciences, Gonabad, Iran

3. Department of Health in Disasters and Emergencies, School of Management and Medical Informatics, Shiraz University of Medical Sciences, Shiraz, Iran

4. Clinical Education Research Center, Shiraz University of Medical Sciences, Shiraz, Iran

5. Health Human Resources Research Center, Shiraz University of Medical Sciences, Shiraz, Iran

\section{Correspondence: \\ Milad Ahmadi Marzaleh \\ Department of Health in Disasters and Emergencies \\ School of Management and Medical Informatics \\ Shiraz University of Medical Sciences, Shiraz, Iran \\ E-mail: miladahmadimarzaleh@yahoo.com}

Conflicts of interest/funding: The authors have no conflict of interest to declare.

Keywords: COVID-19; health; helicopter; pandemic; standard operating procedure

\section{Abbreviations:}

COVID-19: coronavirus disease 2019

EMS: Emergency Medical Services

Received: July 31, 2021

Revised: August 11, 2021

Accepted: August 19, 2021

\section{doi:10.1017/S1049023X21001096}

(C) The Author(s), 2021. Published by Cambridge University Press on behalf of the World Association for Disaster and Emergency Medicine.
Esmaeilzadeh MH, Peyravi M, Ahmadi Marzaleh M. Application of a standard operating procedure in transporting patients during the COVID-19 pandemic in the air Emergency Medical Services. Prehosp Disaster Med. 2021;36(6):807-808.

Dear Editor,

Following the coronavirus disease 2019 (COVID-19) pandemic since December 2019, unforeseen challenges have arisen in the world that threaten the traditional health care delivery processes. One of these challenges is providing emergency services to the patients who are transported by indoor air ambulance services. ${ }^{1,2}$ Due to the lack of information about these patients' infectious conditions and the limited space of the emergency helicopters, the risk of disease transmission is higher for the members of the air emergency medical crew compared to other providers in hospitals or other medical departments. The risk of virus transmission also increases due to the nature of the services provided in the prehospital environment, the acute condition of most patients in this phase, and many medical procedures such as airway management and ventilation associated with aerosol production. ${ }^{3,4}$

Following the outbreak of COVID-19 in many countries, standard operating procedures for infection prevention measures tailored to specific circumstances have been proposed based on the current recommendations of associations and national medical authorities. One of these methods is the wide-spread use of isolated patient compartments in many countries providing helicopter Emergency Medical Services (EMS) to effectively protect the medical crew, which eliminates the need for disinfection of patient cabins and increases the time to access critical helicopter EMS. In Switzerland, simulation training was used as another strategy to prevent the transmission of infection in a recent pandemic and other possible future pandemics in air rescue settings. ${ }^{1,3}$

Other strategies, such as keeping a distance of at least two meters from the patient, reducing the number of health care providers in the patient cabin, and using standard personal protective equipment (PPE), are operational on the agenda of these centers. Furthermore, some European air emergency service providers have taken a major step towards maintaining the health of air emergency staff by allocating designated air relief centers equipped with an isolated infectious disease ward to transport COVID-19approved patients. ${ }^{3}$

\section{Conclusion}

Protection of the physical and mental health of the air emergency medical staff, as the main priority of health care organizations, ${ }^{2}$ is possible via gaining the experiences of the flight crews involved in this issue, as well as the successful experiences of others. Countries have taken measures to implement the existing standards to improve the safety and health of this group of health system employees in Iran. By modeling and localizing the experiences of other successful countries, development of guidelines and special instructions for air emergencies in the Iranian health system can help make health services safer.

Acknowledgement

The authors would like to thank Ms. A. Keivanshekouh at the Research Consultation Center (RCC) of Shiraz University of Medical Sciences for improving the use of English in the manuscript. 


\section{References}

1. Albrecht R, Knapp J, Theiler L, Eder M, Pietsch U. Transport of COVID-19 and other highly contagious patients by helicopter and fixed-wing air ambulance: a narrative review and experience of the Swiss air rescue Rega. Scand J Trauma Resusc Emerg Med. 2020;28(1):40.

2. Tien H, Sawadsky B, Lewell M, Peddle M, Durham W. Critical care transport in the time of COVID-19. CJEM. 2020;22(S2):S84-S88.
3. Hilbert-Carius P, Braun J, Abu-Zidan F, et al. Prehospital care and interfacility transport of 385 COVID-19 emergency patients: an air ambulance perspective. Scand J Trauma Resusc Emerg Med. 2020;28(1):10.

4. Spoelder EJ, Tacken MC, van Geffen G-J, Slagt C. Helicopter transport of critical care COVID-19 patients in the Netherlands: protection against COVID-19 exposure-a challenge to critical care retrieval personnel in a novel operation. Scand J Trauma Resusc Emerg Med. 2021;29(1):41. 